\title{
Tisa: A Language Design and Modular Verification Technique for Temporal Policies in Web Services ${ }^{\star}$
}

\author{
Hridesh Rajan ${ }^{1}$, Jia Tao ${ }^{1}$, Steve Shaner ${ }^{1}$, and Gary T. Leavens ${ }^{2}$ \\ 1 Iowa State University, Ames, Iowa, USA \\ \{hridesh, jtao, smshaner\} @iastate.edu \\ 2 University of Central Florida, Orlando, Florida, USA \\ leavens@eecs.ucf.edu
}

\begin{abstract}
Web services are distributed software components, that are decoupled from each other using interfaces with specified functional behaviors. However, such behavioral specifications are insufficient to demonstrate compliance with certain temporal non-functional policies. An example is demonstrating that a patient's health-related query sent to a health care service is answered only by a doctor (and not by a secretary). Demonstrating compliance with such policies is important for satisfying governmental privacy regulations. It is often necessary to expose the internals of the web service implementation for demonstrating such compliance, which may compromise modularity. In this work, we provide a language design that enables such demonstrations, while hiding majority of the service's source code. The key idea is to use greybox specifications to allow service providers to selectively hide and expose parts of their implementation. The overall problem of showing compliance is then reduced to two subproblems: whether the desired properties are satisfied by the service's greybox specification, and whether this greybox specification is satisfied by the service's implementation. We specify policies using LTL and solve the first problem by model checking. We solve the second problem by refinement techniques.
\end{abstract}

\section{Introduction}

Web services promote abstraction, loose coupling and interoperability of clients and services [1]. The key idea of web services is to introduce a published interface (often a description written in an XML-based language such as WSDL [2]), for communication between services and clients [1]. By allowing components to be decoupled using a specified interface, web services enable platform-independent integration.

Behavioral Contracts for Web Services. A behavioral contract for a web service specifies, for each of the web service's methods the relationships between its inputs and outputs. Such a contract treats the implementation of the service as a black box, hiding all the service's internal states from its clients. The benefit of this encapsulation is that clients do not depend upon the service's changeable design decisions. To illustrate, consider a healthcare service that allows patients to make appointments and ask prescription and health-related questions from healthcare practioners [3].

\footnotetext{
* Rajan and Tao were supported in part by the NSF grant CNS 06-27354. Rajan, Shaner and Leavens were supported in part by the NSF grant CNS 08-08913.
} 
An example JML-like contract [4] for such a service follows.

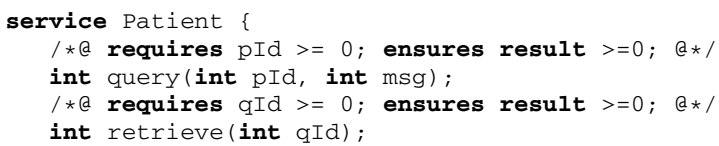

The service description in this contract is written in a form similar to our language, Tisa, to make comparisons easier. It specifies that a service named Patient makes two web-methods available: query and retrieve. The query method takes a patient identifier and a message as arguments. The message is represented as an integer for simplicity (think of it as an index into a table of pre-defined questions, such as "does the test show I have AIDS?"). The precondition of calling this web-method is that the patient identifier is positive; the postcondition is that it returns a positive result. The retrieve method takes a query identifier as argument; its precondition is that this identifier must be positive. Its postcondition is that the result is also positive. These contracts could be checked by observing the interface of the web-methods [567789].

Demonstrating Compliance to Temporal Policies. Let us now consider the following policy inspired from Barth et al.'s work [3]: "a health question about a patient should only be answered by the doctor", "furthermore such answers should only be disclosed to the concerned patients". We will refer to these as "HIPAA policies" as they are similar to regulations in the US health insurance portability and accountability act (HIPAA). The behavioral contract above is insufficient for demonstrating compliance with the HIPAA policies, as it does not provide sufficient details about the internal state of the service. For example, the entity that is finally receiving the query is hidden by query's contract. Demonstrating compliance to such policies is important. In our example, a patient may feel much better about their queries regarding an AIDS test result, if such compliances were demonstrated by the service.

Compliance and Modularity at Conflict. Alternatively suppose the implementation of the two web-methods query and retrieve were available, including the component services that they use. Then demonstrating compliance to the two HIPAA policies would be equivalent to ensuring that the implementation avoids non-compliant states. However, by making code for these methods available, clients might write code that depends on implementation design decisions. As a result, changing these design decisions will become harder, as these changes could break client's code [10].

We thus believe that, for web services, modularity [10] and verification of temporal policies are fundamentally in conflict. To make the service implementation evolvable, modularity requires hiding the design decisions that are likely to change. But to demonstrate compliance to key temporal policies, internal states need to be exposed.

A Language Design and Verification Logic. To reconcile these requirements, we propose a technique based on greybox specifications [11] that exposes only some internal states. This technique enables web service providers to demonstrate compliance to temporal policies, such that above, by exposing only parts of their implementation. A client can verify that the service complies with the desired policies by inspecting a greybox 


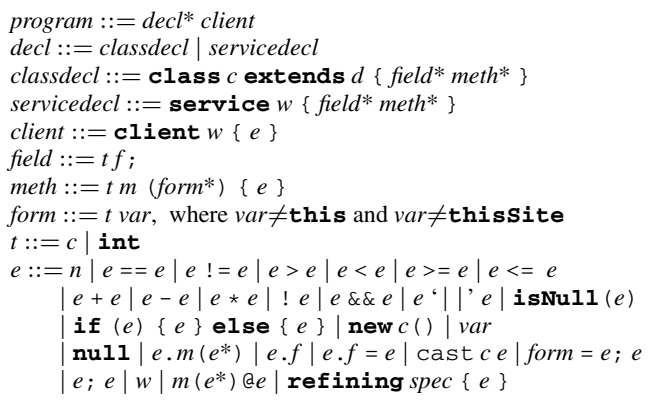

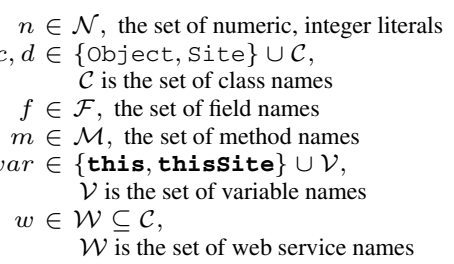

Fig. 2. Abstract syntax, based on [25. Figure 3.1, 3.7]

that the composition of these two verification techniques, applied modularly by clients and all service providers, implies that the web service implementation satisfies the specified temporal policies. In practice, some additional technique, such as proof-carrying code [16], or a hardware-based root of trust [17|18] would be needed to satisfy clients that web services in fact satisfy their specifications.

\section{Tisa Language Design}

In this section, we describe Tisa, an object-oriented (OO) language that incorporates ideas from existing work on specification languages, web services authentication languages and modeling languages. In particular, Tisa's design is inspired by Argus [19] and the work of Gordon and Pucella [20]. (Furthermore, some of our descriptions of the language syntax are adapted from Ptolemy [21].) Tisa is a distributed programming language with statically created web services and a single client, each of which has its own address space. Web services are named and declare web-methods, which can be called by the client and by other services. As a small, core language, the technical presentation of Tisa shares much in common with MiniMAO $_{1}$ [22], a variant of Featherweight Java [23] and Classic Java [24]. Tisa has classes, objects, inheritance, and subtyping, but it does not have super, interfaces, exception handling, built-in value types, privacy modifiers, or abstract methods. Furthermore, other features of web-service description languages (WSDLs) such as composite data types for exchanging messages between services, messages, ports, one-way vs. request-response operations, etc, are omitted to avoid complications in Tisa's theory. However, most of these are syntactic sugars that can be desugared to existing constructs in Tisa. Tisa features new mechanisms for declaring policies and greybox specifications. Our description starts with its programming features, and then describes its specification features.

\subsection{Program Syntax}

The syntax of Tisa executable programs is shown in Figure 2 and explained below. A Tisa program consists of zero or more declarations, and a client (see Figure 3). Declarations are either class declarations or web service declarations. 
specification $::=$ servicespec $^{*}$ servicespec $::=$ service $w\left\{\right.$ wmspec $\left.^{*}\right\}$ wmspec $::=t m\left(\right.$ form $\left.^{*}\right)\{$ se $\}$ form $::=t$ var, where varfthissite spec $::=$ requires $s p$ ensures $s p$

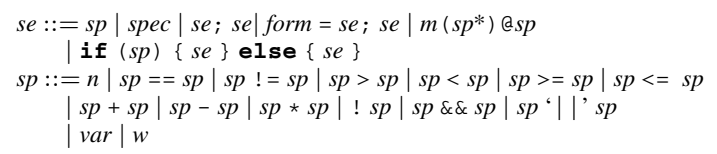

Fig. 4. Syntax for Writing Specifications in Tisa

operations operate on integers, with 0 representing false, and all other integer values representing true. An if $\left(e_{1}\right) \quad\left\{e_{2}\right\}$ else $\left\{e_{3}\right\}$ expression tests if $e_{1}$ is nonzero; if so it returns the value of $e_{2}$, otherwise it returns the value of $e_{3}$.

The standard $\mathrm{OO}$ expressions include object construction (new $c($ ) ), variable dereference (var, including this), field dereference (e.f), null, cast (cast $t e$ ), assignment to a field $\left(e_{1} . f=e_{2}\right)$, sequencing $\left(e_{1} ; e_{2}\right)$, casts and a definition block $(t$ var $=$ $\left.e_{1} ; e_{2}\right)$. The other OO expressions are standard [25|22].

There are three new expressions: web service names, web-method calls, and refining statements. Web service names of form $w$ are constants. A web-method call has the form $\left(m\left(e^{*}\right) @ e_{w}\right)$, where the expression following the at-sign $\left(e_{w}\right)$ denotes the name of the web service name that will execute the web-method call named $m$ with formals $e^{*}$. A refining statement, of the form refining spec $\{e\}$, is used in implementing Tisa's greybox specifications (see below). It executes the expression $e$, which is supposed to satisfy the specification spec.

\subsection{Specification Constructs}

The syntax for writing specifications in Tisa is shown in Figure 4. In this figure, all nonterminals that are used but not defined are the same as in Figure 2 Specifications consist of several service specifications (servicespec). (Since we only permit integers to be sent to and returned from web-method calls, we omit class declarations from specifications.) A service specification may contain finite number of web-method specifications (wmspec). All fields are hidden, so field declarations are not allowed in a service specification. The body of a web-method specification contains a side-effect free expression (se). Many expressions from Figure 2 also appear as such side-effect free expressions, but not field-related operations, method calls, and isNul1. Web-method call expressions are allowed and so are local variable definition expressions.

The main new feature of specifications, borrowed from the refinement calculus and the greybox approach, is the specification expression (spec). Such an expression hides (abstracts from) a piece of code in a correct implementation. The most general form of specification expression is requires $s p_{1}$ ensures $s p_{2}$, where $s p_{1}$ is a precondition expression and $s p_{2}$ is a postcondition. Such a specification expression hides program details by specifying that a correct implementation contains a refining expression whose body expression, when started in a state that satisfies $s p_{1}$, will terminate in a state that satisfies $s p_{2}[15]$.

In examples we use two sugared forms of specification expression. The expression preserve $s p$ is sugar for requires $s p$ ensures $s p$ and establish $s p$ is sugar for requires 1 ensures $s p$.

An example greybox specification of the web service Patient appears in Figure 1 The specification of the web-method query appears on line 26, and specifies (and 
thus exposes) all the code for that method. The specification of retrieve hides a bit more in its preserve expression (line 29). But it also exposes code that makes a web-method call retrieve to the Secretary or Doctor. With these greybox specifications, enough details are exposed about what the service does when invoking other services, which makes it feasible to show compliance to the HIPAA policies.

\subsection{Constructs for Specifying Policies}

Our simple policy specification language is similar to Linear Temporal Logic [14].

$\Phi($ specification $)::=\mathcal{P}$ (specification) $|\neg \phi| \phi_{1} \wedge \phi_{2}\left|\phi_{1} \mathbf{U} \phi_{2}\right| \mathbf{X} \phi$

The language specifies histories that are sequences of web method calls. For a given specification, a policy can be an atomic proposition in $\mathcal{P}$ (specification); a negation of a policy or boolean combination of policies. For simplicity here we take the set of legal propositions $\mathcal{P}$ (specification) to be all legal web-method calls in the given specification. This set can be statically computed from the specification against which the policy is to be verified by traversing the abstract syntax tree of the specification up to the depth of web-method specifications. The operator $\mathbf{U}$ is read as "until" and $\mathbf{X}$ as "next." $\phi_{1} \mathbf{U} \phi_{2}$ states that policy $\phi_{2}$ must be satisfied after policy $\phi_{1}$ is satisfied along all executions of the service. $\mathbf{X} \phi$ states that policy $\phi$ must be satisfied in the next state (i.e., at the next web method call). We also use the following common abbreviations:

$$
\begin{array}{lll}
\phi_{1} \vee \phi_{2} \equiv \neg\left(\neg \phi_{1} \wedge \neg \phi_{2}\right) & \phi_{1} \rightarrow \phi_{2} \equiv \neg \phi_{1} \vee \phi_{2} & \text { true } \equiv \phi \vee \neg \phi \\
\text { false } \equiv \neg \text { true } & \mathbf{F} \phi \equiv \text { true } \mathbf{U} \phi & \mathbf{G} \phi \equiv \neg \mathbf{F} \neg \phi
\end{array}
$$

The constant true means that the service does not have any obligation. The operator $\mathbf{F}$ is read as "eventually" and $\mathbf{G}$ as "always". Below we present two sample policies for our healthcare service example.

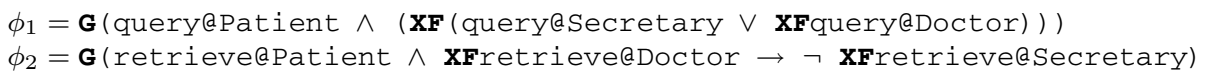

The policy $\phi_{1}$ states that whenever there is a web-method call query@Patient, there is eventually a web-method call query at one of the sites Secretary or Doctor. This policy says that a query is eventually delivered to one of the healthcare providers. The policy $\phi_{2}$ encodes the constraint that a health answer that comes from doctors goes directly to the patient, and is never forwarded to secretaries. In terms of the service specification, if there is a web-method call retrieve@Patient and it is followed by a web-method call retrieve@Doctor, then there is never a web-method call retrieve at the site Secretary in the same trace.

\subsection{Dynamic Semantics of Tisa's Constructs}

This section defines a small step operational semantics for Tisa programs (adapted from Clifton's work [25]). In the semantics, all declarations are formed into a single class table that maps class names and web service names to class and service declarations, respectively. However, despite this global view of declarations, the model of storage is distributed, with each web service having an independent store. 

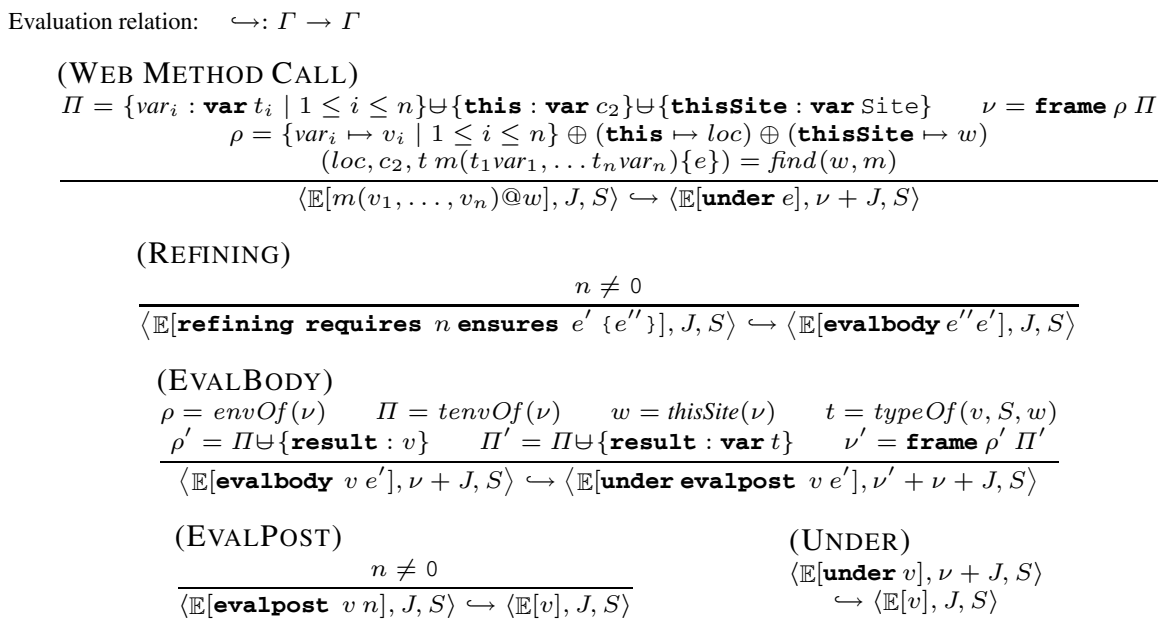

Fig. 5. Operational semantics of Tisa. Standard OO rules are omitted.

The operational semantics relies on four expressions, not part of Tisa's surface syntax, to record final or intermediate states of the computation. The loc expression represents locations in the store. The under expression is used as a way to mark when the evaluation stack needs popping. The evalbody and evalpost are used in evaluation of specification expressions. The three exceptions NullPointerException, ClassCastException, and SpecException record various problems orthogonal to the type system.

A configuration in the semantics contains an expression $(e)$, an evaluation stack $(J)$, and a store $(S)$. The current web service name is maintained in the evaluation stack under the name thissite. The auxiliary function thisSite extracts the current web service name from a stack frame. Stacks are an ordered list of frames, each frame recording the static environment, $\rho$, and a type environment. (The type environment, $\Pi$, is only used in the type soundness proof.) The static environment $\rho$ maps identifiers to values. A value is a number, a web service name (site), a location, or null. Stores are maps from locations to storable values, which are object records. Object records have a class and also a map from field names to values.

The semantics is presented as a set of evaluation contexts $\mathbb{E}$ and an one-step reduction relation [26] that acts on the position in the overall expression identified by the evaluation context as shown in Figure 5 Standard OO rules are presented in our technical report [27]. The key rule is (WEB METHOD CALL), which uses the auxiliary function find to retrieve the body of the web method from a class table $C T$ implicitly used by the semantics. It creates the frame for execution of the web method with necessary static environment and type environment and starts execution of the web method body. The under $e$ expression is used in the resulting configuration to mark that the stack should be popped when the evaluation of $e$ is finished.

Evaluation of a refining expression involves 3 steps. First the precondition is evaluated (due to the context rules). If the precondition is non-zero (i.e., true), then the next configuration is evalbody $e^{\prime \prime} e^{\prime}$, where $e^{\prime \prime}$ is the body and $e^{\prime}$ is the postcondition 
(regarded as an expression). The body is then evaluated; if it yields a value $v$, then the next configuration is under evalpost $v e^{\prime}$, with a new stack frame that binds result to $v$ pushed on the stack. The type of result in the type environment $\Pi^{\prime}$ is determined by the auxiliary function type Of. Finally, the (EVALPOST) rule checks that the postcondition is true and uses the body's value as the value of the expression.

\section{Verification of Policies in Tisa}

A key contribution of our work is to decouple, with Tisa's language design, the verification of whether a policy is satisfied by a web service implementation into two verification tasks that can proceed modularly and independently. The first task is to verify whether a policy is satisfied by the service specification. The second task is to verify whether the service specification is satisfied by the service implementation. Three benefits follow from this modular approach. First, the service implementation need not be visible to clients, as a client uses the specification to determine whether their desired policies hold. Thus, our approach achieves modularity for service implementations. Second, regardless of the number of clients, the second verification task must only be done once; thus our approach is likely to be scalable for web service providers. Last but not the least, policy verification is performed on the (generally smaller) specification. Thus, our approach has efficiency benefits for policy verification.

Determining whether a policy is satisfied by the specification can be reduced to a standard model checking problem [14]. We claim no contribution here; rather, the novelty of our approach is in a combination of these two techniques, enabled by a careful language design. To show the feasibility of applying ideas from model checking [14] and refinement calculus [12 13] to our problem, in the rest of this section we describe our techniques for verifying policies and refinement.

\subsection{Verifying Policies}

We adopt the standard automata-theoretic approach for verifying linear temporal logic formulas proposed by Vardi and Wolper [28] to verify policies in Tisa. Following Vardi and Wolper [28], a policy $\phi \in \Phi(\mathcal{S})$ is viewed as a finite-state acceptor and a specification $\mathcal{S}$ as a finite-state generator of expression execution histories. Thus the specification $\mathcal{S}$ satisfies policy $\phi$ if every (potentially infinite) history generated by $\mathcal{S}$ is accepted by $\phi$, in other words, if $\mathcal{S} \cap \neg \phi$ is empty.

Figure 6 shows main parts of an algorithm for constructing a finite-state machine $\mathcal{F}(\mathcal{S})=\left(\mathcal{Z}, z_{0}, R, \Delta\right)$ from a Tisa specification $\mathcal{S}$. Here, $Z$ is a finite set of states, $z_{0}$ is the initial state, $R$ is a total accessibility relation, $\Delta: Z \rightarrow 2^{\mathcal{P}(\mathcal{S})}$, which determines how truth values are assigned to propositions in each state [28, pp. 5]. All rules make use of unions for joining set of states $(Z)$ and disjoint union $(\uplus)$ for joining propositions. Rules for standard OO expressions are omitted.

The (IF EXP FSM) rule demonstrates creation of non-deterministic transitions in the state machine. It computes the FSMs corresponding to the true branch and the false branch of the if expression with initial states $z^{\prime}$ and $z^{\prime \prime}$ and joins these two FSMs to make a new FSM with initial state $z$. Corresponding to the state $z^{\prime}$, which corresponds 
Production relation: $\quad N T \vdash \operatorname{se} \rightsquigarrow\left(Z, z_{0}, R, \Delta\right), N T \quad$ where $N T \in \mathcal{N} \mathcal{T}=\mathcal{W} \times \mathcal{M} \rightarrow Z$

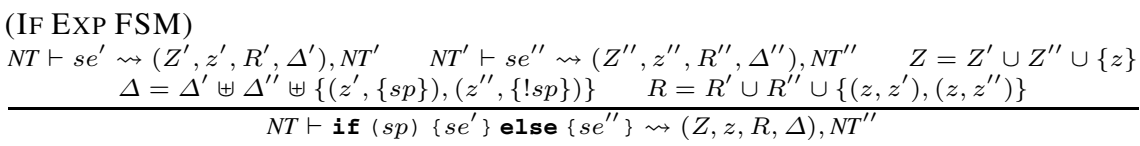

(Web Method Call FSM 1)

$\begin{array}{cr}\neg(\exists z:: N T(w, m)=z) \\ N T^{\prime}=N T \cup((w, m), z) \quad m\left(t_{1}, \ldots t_{n}\right)\{s e\}=f i n d(w, m) & N T^{\prime} \vdash s e \rightsquigarrow\left(Z^{\prime}, z^{\prime}, R^{\prime}, \Delta^{\prime}\right), N T^{\prime \prime} \\ Z=Z^{\prime} \cup\{z\} \quad \Delta=\Delta^{\prime} \uplus\left\{\left(z^{\prime},\{m @ w\}\right)\right\} & R=R^{\prime} \cup\left\{\left(z, z^{\prime}\right)\right\}\end{array}$

(Web Method Call FSM 2)

$\frac{z=N T(w, m)}{N T \vdash m\left(v_{1}, \ldots, v_{n}\right) @ w \rightsquigarrow(\{z\}, z,\{\},\{\}), N T}$

(SPEC EXP FSM)

$Z=\left\{z_{1}, z_{2}, z_{3}, z_{4}\right\} \quad R=\left\{\left(z, z_{1}\right),\left(z, z_{2}\right),\left(z_{1}, z_{3}\right),\left(z_{1}, z_{4}\right),\left(z_{3}, z^{\prime}\right)\right\}$
$\frac{\Delta_{\text {pre }}=\left\{\left(z_{1},\left\{s p_{1}\right\}\right),\left(z_{2},\left\{! s p_{1}\right\}\right)\right\} \quad \Delta=\Delta_{p r e} \uplus\left\{\left(z_{3},\left\{s p_{1}, s p_{2}\right\}\right),\left(z_{4},\left\{s p_{1}, ! s p_{2}\right\}\right)\right\}}{N T \vdash \text { requires } s p_{1} \text { ensures } s p_{2} \rightsquigarrow(Z, z, R, \Delta), N T}$

Fig. 6. Finite-state machine construction, built from expressions in a specification

to the true branch, the proposition $s p$ is added to $\Delta$, which corresponds to the conditional expression evaluating to the truth value true. Similarly for the state $z^{\prime \prime}$, which corresponds to the false branch, the proposition $! s p$ is added to $\Delta$, which corresponds to the conditional expression evaluating to the truth value false.

The (SPEC EXP FSM) rule models the cases for satisfaction of precondition and postcondition. The (Web Method CALL FSM) rules make use of a table NT that maps pairs of web service names and method names $(w, m)$ to states. This table is used to account for recursion in web-method calls. Finally, the finite-state machine for a service specification is created by first creating finite-state machines for each of its web-method specifications as if it is being called and by joining them using an extra state that becomes the new initial state.

Given the $\operatorname{FSM} \mathcal{F}(\mathcal{S})$ we construct a Büchi automaton [29], $\mathcal{B}(\neg \phi)$ for the policy $\phi \in \Phi(\mathcal{S})$ as shown by Vardi and Wolper [28]. Specification $\mathcal{S}$ satisfies the policy $\phi$ if $\mathcal{F}(\mathcal{S}) \cap \mathcal{B}(\neg \phi)$ is empty.

\subsection{Verifying Refinement}

Our technique for checking whether a program refines a specification in Tisa is similar to the work of Shaner, Leavens and Naumann [15]. An implementation refines a specification if it meets two criteria: first, that the code and specification are structurally similar and second, that the body of every refining expression obeys the specification it is refining. By structural similarity we mean that for every non-specification expression in the specification, the implementation has the identical expression at that position in the code. This is checked in a top-down manner as shown in Figure 7 The operational semantics rules (REFINING), (EVALBODY) and (EVALPOST) ensure that the body of every refining expression obeys the specification it is refining. 
(PROGRAM REF)

$\forall i \in\{1 \ldots m\} \exists j \in\{1 \ldots n\}$ decl $_{j} \in{\text { servicedecl } \wedge \text { servicespec }_{i} \sqsubseteq \text { decl }_{j}}$

servicespec $_{1} \ldots$ servicespec $_{m} \sqsubseteq$ decl $_{1} \ldots$ decl $_{n}$

(SERVICE REF)

$\forall i \in\{1 . . m\} \exists j \in\{1 \ldots n\}$ wmspec $_{i} \sqsubseteq$ meth $_{j}$

service $w\left\{\right.$ wmspec $_{1} \ldots$ wmspec $\left._{n}\right\}$
$\sqsubseteq$ service $w\left\{\right.$ field $_{1} \ldots$ field $_{f}$ meth $_{1} \ldots$ meth $\left._{n}\right\}$

(Web Method Ref)

tm(form fof $_{1} \ldots$ form $\left._{n}\right)\{\mathrm{se}\}$

$\sqsubseteq t m\left(\right.$ form $_{1} \ldots$ form $\left._{n}\right)\{e\}$

$($ SEQ EXP REF)
$\frac{s e_{1} \sqsubseteq e_{1} s e_{2} \sqsubseteq e_{2}}{s e_{1} ; s e_{2}}$
$\sqsubseteq e_{1} ; e_{2}$

(WeBCALl EXP ReF)

$\frac{\left(\forall i \in\{1 . . n\}:: s p_{i} \sqsubseteq e_{i}\right) \quad s p_{w} \sqsubseteq e_{w}}{m\left(s p_{1}, \ldots, s p_{n}\right) @ s p_{w} \sqsubseteq m\left(e_{1}, \ldots, e_{n}\right) @ e_{w}}$

\section{(IF EXP REF)}

$\frac{s p \sqsubseteq e_{b} \quad s e_{T} \sqsubseteq e_{T} \quad s e_{F} \sqsubseteq e_{F}}{\text { if }(s p)\left\{s e_{T}\right\} \text { else }\left\{s e_{F}\right\}}$

(SPEC EXP REF)

(requires $s p_{1}$ ensures $s p_{2}$ ) $=s p e c$
(DEF EXP REF)

$\frac{s p \sqsubseteq e_{\text {init }} \quad s e \sqsubseteq e_{\text {body }}}{\text { form }=s p ; s e}$
$\sqsubseteq$ form $=e_{\text {init }} ; e_{\text {body }}$

Fig. 7. Inference rules for proving Tisa refinement

\subsection{Soundness of Verification Technique}

The proof of soundness of our verification technique uses the following three definitions.

Definition 1 (A Path for $\mathcal{S}$ ). Let $\mathcal{S}$ be a specification and $\mathcal{F}(\mathcal{S})=\left(\mathcal{Z}, z_{0}, R, \Delta\right)$ be the FSM for $\mathcal{S}$ constructed using algorithm shown in Figure 6 a path $t$ for $\mathcal{S}$ is a (possibly infinite) sequence of pairs $\left(z_{i}, \Delta\left(z_{i}\right)\right)$ starting with pair $\left(z_{0}, \Delta\left(z_{0}\right)\right)$, where for each $i \geq 0, z_{i} \in Z$ and $\left(z_{i}, z_{i+1}\right) \in R$.

Definition 2 (A Path for $P$ ). Let $P$ be a program and $\mathcal{C F} \mathcal{G}(P)=\left(Z^{\prime}, z_{0}^{\prime}, R^{\prime}, \Delta^{\prime}\right)$ be an annotated control flow graph for $P$, where $Z^{\prime}$ is the set of nodes representing expressions in program, $R^{\prime}$ is the control flow relation between nodes, and $\Delta^{\prime}: Z^{\prime} \rightarrow$ $2^{\mathcal{P}(P)}$ is such that for each $z_{i}^{\prime} \in Z^{\prime}$, if it represents a web-method call expression $m(.) @$.$w then \left(z_{i}^{\prime},\{m @ w\}\right) \in \Delta^{\prime}$. A path $t^{\prime}$ for $P$ is a (possibly infinite) sequence of pairs $\left(z_{i}^{\prime}, \Delta\left(z_{i}^{\prime}\right)\right)$ starting with pair $\left(z_{0}^{\prime}, \Delta\left(z_{0}^{\prime}\right)\right)$, where for each $i \geq 0, z_{i}^{\prime} \in Z$ and $\left(z_{i}^{\prime}, z_{i+1}^{\prime}\right) \in R^{\prime}$.

Definition 3 (Path Refinement). Let $t$ be a path for $\mathcal{S}$ and $t^{\prime}$ be a path for $P$. Then $t$ is refined by $t^{\prime}$, written $t \sqsubseteq t^{\prime}$, just when one of the following holds:

- $t \equiv t^{\prime}$ i.e., for each $i \geq 0,\left(z_{i}, \delta_{i}\right) \in t$ and $\left(z_{i}^{\prime}, \delta_{i}^{\prime}\right) \in t^{\prime}$ implies $z_{i}=z_{i}^{\prime}$ and $\delta_{i}=\delta_{i}^{\prime}$,

- $t=(z, \delta)+t_{1}$ and $t^{\prime}=\left(z^{\prime}, \delta^{\prime}\right)+t_{1}^{\prime}$ and $\delta \Rightarrow \delta^{\prime}$ and $t_{1} \sqsubseteq t_{1}^{\prime}$,

- $t=(z, \delta)+t_{1}$ and $t^{\prime}=\left(z_{1}^{\prime}, \delta_{1}^{\prime}\right)+\ldots+\left(z_{n}^{\prime}, \delta_{n}^{\prime}\right)+t_{1}^{\prime}$ and $\delta \Rightarrow\left(\delta_{1}^{\prime} \uplus \ldots \uplus \delta_{n}^{\prime}\right)$ and $t_{1} \sqsubseteq t_{1}^{\prime}$, or

- $t=t_{1}+t_{2}$ and $t^{\prime}=t_{1}^{\prime}+t_{2}^{\prime}$ and $t_{1} \sqsubseteq t_{1}^{\prime}$ and $t_{2} \sqsubseteq t_{2}^{\prime}$.

Lemma 1. Let $P \in$ program and $\mathcal{S} \in$ specification be given. If $P$ refines $\mathcal{S}$, then for each path $t^{\prime}$ for $P$ there exists a path $t$ for $\mathcal{S}$ such that $t \sqsubseteq t^{\prime}$.

Proof Sketch: The proof for this lemma follows from structural induction on the refinement rules shown in Figure 7 . Details are contained in our technical report [27]. 
Lemma 2. Given a specification $\mathcal{S}$ and a policy $\phi \in \Phi(\mathcal{S})$, the automaton $\mathcal{F}(\mathcal{S}) \cap$ $\mathcal{B}(\neg \phi)$ accepts a language, which is empty when the specification satisfies the policy.

The proof of this lemma follows from standard proofs in model checking, in particular, from Lemma 3.1, Theorem 2.1 and Theorem 3.3. given by Vardi and Wolper [28, pp. 4,6]. Details are contained in our technical report [27].

Theorem 1. Let $\mathcal{S}$ be a specification, $\phi$ be a policy in $\Phi(\mathcal{S})$, and $P$ be a program. Let $\phi$ be satisfied by the specification $\mathcal{S}$ and $P$ be a refinement of $\mathcal{S}$ (as defined in Figure 7 ). Then the policy $\phi$ is satisfied by the program $P$.

Proof Sketch: The proof follows from lemma 1 and 2 From lemma 1 we have that each path in the program refines a path in the specification. From lemma 2 and the assumptions of this theorem, we have that $\phi$ is satisfied on all paths in $\mathcal{S}$. Thus, $\phi$, which is written over $\mathcal{P}(\mathcal{S})$, is also satisfied for $P$.

\section{Related Work}

In this section, we discuss techniques that are closely related to our approach.

Greybox specifications. We are not the first to consider greybox specifications [11] as a solution for verification problems. Barnett and Schulte [30|31] have considered using greybox specifications written in AsmL [32] for verifying contracts for .NET framework. Wasserman and Blum [33] also use a restricted form of greybox specifications for verification. Tyler and Soundarajan [34] and most recently Shaner, Leavens, and Naumann [15] have used greybox specifications for verification of methods that make mandatory calls to other dynamically-dispatched methods. Compared to these related ideas, to the best of our knowledge our work is the first to consider greybox specifications as a mechanism to decouple verification of web services without exposing all of their implementation details. Secondly, most of these, e.g. Shaner, Leavens, and Naumann [15] use the refinement of Hoare logic as their underlying foundation. This was insufficient to tackle the problem that we address, which required showing refinement of (a variant of) linear temporal logic. Thus adaptation of much of their work was not possible, although we were able to adapt the notion of structural refinement.

Specification and Verification Techniques for Web Services. The technique proposed by Bravetti and Zavattaro [35] for determining whether the behavioral contract of a service correctly refines its desired requirements in a composition of web-services is closely related and complementary to this work. The main difference between this work and the current work is that we verify refinement of greybox specifications by service implementations that allows us to reason about temporal policies, while hiding much of the implementation. However, we foresee a combination of our work and Bravetti and Zavattaro's work for determining fitness of a service implementation in a desired composition of web-services.

Some approaches have recently been proposed to verify contracts for web services, as seen in the works of Acciai and Boreale [36], Kuo et al. [8], Baresi et al. [6], Barbon et al. [5], etc. These ideas focus on verifying the behavioral contracts as defined by the externally visible interface of the web services, whereas our work provides a 
principled, modular technique for verifying such policies that require inspecting the web service implementation to a limited extent.

Castagna, Gesbert and Padovani present a formalism for specifying web services based on the notion of "filtering" the possible behaviors of an existing web service to conform to the behavior of some contract [7]. These filters take the form of coercions that limit when and how an available service may be consumed. These coercions permits contract subtyping and support reasoning in a language-independent way about the sequence of reads and writes performed between service clients and providers. Their contracts are intended to constrain the usage scenarios of a web service, whereas the present work describes a modular way to specify the observable behaviors that occur inside service implementations.

Bartoletti et al. [37] provide a formalization of web service composition in order to reason about the security properties provided by connected services. While they ignore policy language details, our work shows how the amount of overhead used to relate specifications to policies depends on the level of detail in the policy language. Furthermore, we believe greybox reasoning grants real benefits in readability and modularity over their type system. We view later work developing executable specifications for design of web services [38] as possible future work for Tisa.

Another approach [39] proposes an architecture to enforce these access policies at component web services, but again the work is tightly coupled to the WS-SensFlow and Axis implementations. Srivatsa et al. [40] propose an Access Control system for composite services which does not take care of the Trust in the resulting service oriented architecture. Skalka and Wang [41] introduced a trust but verify framework which is an access control system for web services, but they do not provide temporal reasoning for the verification of policies. By recording the sequence of program events in temporal order, Skalka and Smith [42] are able to verify the policies such as whether the events were happened in a reasonable order, but the mechanism does not support decoupling the model and the implementation. Other approaches [4344] either do not have a formal model supporting them or are tightly coupled with implementations.

\section{Future Work and Conclusions}

We have designed Tisa to be a small core language to clearly communicate how it allows users to balance compliance and modularity in web service specification. However, our desire for simplicity and clarity led us to leave for future work many practical and useful extensions. The most important future work in the area of Tisa's semantics is to investigate refinement of information flow properties. It would also be interesting to investigate the utility of Tisa's specification forms for reasoning about the composition of web services.

Verifying web services is an important problem [7/568,9], which is crucial for wider adoption of this improved modularization technique that enables new integration possibilities. There are several techniques for verifying web-services using behavioral interfaces, but none facilitates verification that requires access to internal states of the service. To that end, the key contribution of this work is to identify the conflict between verification of temporal properties and modularity requirements in web services. Our language design, Tisa, addresses these challenges. It allows service providers to demonstrate 
compliance to policies expressed in an LTL-like language [14]. We also showed that policies in Tisa can be verified by clients using just the specification. Furthermore, refinement of specifications by program ensures that conclusion drawn from verifying policies are valid for Tisa programs. Another key benefit of Tisa is that its greybox specifications [11] allow service providers to encapsulate changeable implementation details by hiding them using a combination of spec and refining expressions. Thus, Tisa provides significant modularity benefits while balancing the verification needs.

\section{References}

1. Papazoglou, M.P., Georgakopoulos, D.: Service-oriented computing: Introduction. Commun. ACM 46(10), 24-28 (2003)

2. Christensen, E., Curbera, F., Meredith, G., Weerawarana, S.: Web services description language (WSDL) 1.1. Technical report, World Wide Web Consortium (March 2001)

3. Barth, A., Mitchell, J., Datta, A., Sundaram, S.: Privacy and utility in business processes. In: CSF 2007, pp. 279-294 (2007)

4. Leavens, G.T., Baker, A.L., Ruby, C.: Preliminary design of JML: a behavioral interface specification language for Java. SIGSOFT Softw. Eng. Notes 31(3), 1-38 (2006)

5. Barbon, F., Traverso, P., Pistore, M., Trainotti, M.: Run-time monitoring of instances and classes of web service compositions. In: ICWS 2006, pp. 63-71 (2006)

6. Baresi, L., Ghezzi, C., Guinea, S.: Smart monitors for composed services. In: ICSOC 2004, pp. 193-202 (2004)

7. Castagna, G., Gesbert, N., Padovani, L.: A theory of contracts for web services. In: POPL 2008, pp. 261-272 (2008)

8. Kuo, D., Fekete, A., Greenfield, P., Nepal, S., Zic, J., Parastatidis, S., Webber, J.: Expressing and reasoning about service contracts in service-oriented computing. In: ICWS 2006, pp. 915-918 (2006)

9. Wada, H., Suzuki, J., Oba, K.: Modeling non-functional aspects in service oriented architecture. In: IEEE International Conference on Services Computing (SCC 2006), pp. 222-229 (2006)

10. Parnas, D.L.: On the criteria to be used in decomposing systems into modules 15(12), 1053 1058 (1972)

11. Büchi, M., Weck, W.: The greybox approach: When blackbox specifications hide too much. Technical Report 297, Turku Center for Computer Science (August 1999)

12. Back, R.J.R., von Wright, J.: Refinement calculus, part i: sequential nondeterministic programs. In: REX workshop, pp. 42-66 (1990)

13. Morris, J.M.: A theoretical basis for stepwise refinement and the programming calculus. Sci. Comput. Program. 9(3), 287-306 (1987)

14. Edmund, M., Clarke, J., Grumberg, O., Peled, D.A.: Model checking. MIT Press, Cambridge (1999)

15. Shaner, S.M., Leavens, G.T., Naumann, D.A.: Modular verification of higher-order methods with mandatory calls specified by model programs. In: OOPSLA 2007, pp. 351-368 (2007)

16. Necula, G.C.: Proof-carrying code. In: POPL 1997, pp. 106-119 (1997)

17. Rajan, H., Hosamani, M.: Tisa: Towards trustworthy services in a service-oriented architecture. IEEE Transactions on Services Computing (SOC) 1(2) (2008)

18. Hosamani, M., Narayanappa, H., Rajan, H.: How to trust a web service monitor deployed in an untrusted environment? In: NWESP 2007: Proceedings of the Third International Conference on Next Generation Web Services Practices, pp. 79-84 (2007)

19. Liskov, B., Scheifler, R.: Guardians and actions: Linguistic support for robust, distributed programs. TOPLAS 5(3), 381-404 (1983) 
20. Gordon, A.D., Pucella, R.: Validating a web service security abstraction by typing. Formal Aspects of Computing 17(3), 277-318 (2005)

21. Rajan, H., Leavens, G.T.: Ptolemy: A language with quantified typed events. In: Vitek, J. (ed.) ECOOP 2008. LNCS, vol. 5142, pp. 155-179. Springer, Heidelberg (2008)

22. Clifton, C., Leavens, G.T.: MiniMAO 1 : Investigating the semantics of proceed. Science of Computer Programming 63(3), 321-374 (2006)

23. Igarashi, A., Pierce, B., Wadler, P.: Featherweight Java: A minimal core calculus for Java and GJ. In: OOPSLA 1999, pp. 132-146 (1999)

24. Flatt, M., Krishnamurthi, S., Felleisen, M.: A programmer's reduction semantics for classes and mixins. In: Formal Syntax and Semantics of Java, pp. 241-269 (1999)

25. Clifton, C.: A design discipline and language features for modular reasoning in aspectoriented programs. Technical Report 05-15, Iowa State University (Jul 2005)

26. Wright, A.K., Felleisen, M.: A syntactic approach to type soundness. Information and Computation 115(1), 38-94 (1994)

27. Rajan, H., Tao, J., Shaner, S.M., Leavens, G.T.: Reconciling trust and modularity in web services. Technical Report 08-07, Dept. of Computer Sc., Iowa State U. (July 2008)

28. Vardi, M.Y., Wolper, P.: An automata-theoretic approach to automatic program verification. In: Proceedings of the First Symposium on Logic in Computer Science, pp. 322-331 (1986)

29. Buchi, J.: On a decision method in restricted second order arithmetic. In: Proc. Internat. Congr. Logic, Method. and Philos. Sci., pp. 1-12 (1960)

30. Barnett, M., Schulte, W.: Runtime verification of .net contracts. Journal of Systems and Software 65(3), 199-208 (2003)

31. Barnett, M., Schulte, W.: Spying on components: A runtime verification technique. In: Workshop on Specification and Verification of Component-Based Systems (2001)

32. Barnett, M., Schulte, W.: The ABCs of specification: AsmL, Behavior, and Components. Informatica 25(4), 517-526 (2001)

33. Wasserman, H., Blum, M.: Software reliability via run-time result-checking. J. ACM 44(6), 826-849 (1997)

34. Tyler, B., Soundarajan, N.: Black-box testing of grey-box behavior. In: Petrenko, A., Ulrich, A. (eds.) FATES 2003. LNCS, vol. 2931, pp. 1-14. Springer, Heidelberg (2004)

35. Bravetti, M., Zavattaro, G.: Towards a unifying theory for choreography conformance and contract compliance. In: Lumpe, M., Vanderperren, W. (eds.) SC 2007. LNCS, vol. 4829, pp. 34-50. Springer, Heidelberg (2007)

36. Acciai, L., Boreale, M.: XPi: A typed process calculus for XML messaging. Science of Computer Programming 71(2), 110-143 (2008)

37. Bartoletti, M., Degano, P., Ferrari, G.L.: Types and effects for secure service orchestration. In: CSFW, pp. 57-69 (2006)

38. Bartoletti, M., Degano, P., Ferrari, G.L., Zunino, R.: Semantics-based design for secure web services. IEEE Trans. Software Eng. 34(1), 33-49 (2008)

39. Wei, J., Singaravelu, L., Pu, C.: Guarding sensitive information streams through the jungle of composite web services. In: ICWS 2007, pp. 455-462 (2007)

40. Srivatsa, M., Iyengar, A., Mikalsen, T., Rouvellou, I., Yin, J.: An access control system for web service compositions. In: ICWS 2007, pp. 1-8 (2007)

41. Skalka, C., Wang, X.S.: Trust but verify: authorization for web services. In: SWS, pp. 47-55 (2004)

42. Skalka, C., Smith, S.F.: History effects and verification. In: Chin, W.-N. (ed.) APLAS 2004. LNCS, vol. 3302, pp. 107-128. Springer, Heidelberg (2004)

43. Biskup, J., Carminati, B., Ferrari, E., Muller, F., Wortmann, S.: Towards secure execution orders for composite web services. In: ICWS 2007, pp. 489-496 (2007)

44. Vorobiev, A., Han, J.: Specifying dynamic security properties of web service based systems. In: SKG 2006, p. 34 (2006) 